\title{
Differential effects of minocycline on microvascular complications in murine models of type 1 and type 2 diabetes
}

Stephanie A. Eid ${ }^{1 \#}$, Phillipe D. O'Brien ${ }^{1 \#}$, Lucy M. Hinder ${ }^{1}$, John M. Hayes ${ }^{1}$, Faye E. Mendelson ${ }^{1}$, Hongyu Zhang ${ }^{2}$, Samanthi Narayanan ${ }^{1}$, Steven F. Abcouwer ${ }^{3}$, Frank C. Brosius III',4, Subramaniam Pennathur ${ }^{2,4}$, Masha G. Savelieff ${ }^{1}$ and Eva L. Feldman ${ }^{1 *}$

${ }^{1}$ Department of Neurology, University of Michigan, Ann Arbor, MI, 48109, USA

${ }^{2}$ Division of Nephrology, Department of Internal Medicine, University of Michigan, Ann Arbor, MI, 48109, U.S.A.

${ }^{3}$ Department of Ophthalmology and Visual Sciences, University of Michigan, Ann Arbor, MI, 48105, U.S.A.

${ }^{4}$ Departments of Molecular and Integrative Physiology, University of Michigan, Ann Arbor, MI, 48109, U.S.A.

\begin{abstract}
Diabetes is a global healthcare problem associated with enormous healthcare and personal costs. Despite glucose lowering agents that control glycaemia, both type 1 (T1D) and type (T2D) diabetes patients often develop microvascular complications that increase morbidity and mortality. Current interventions rely on careful glycemic control and healthy lifestyle choices, but these are ineffective at reversing or completely preventing the major microvascular complications, diabetic peripheral neuropathy (DPN), diabetic retinopathy (DR), and diabetic kidney disease (DKD). Minocycline, a tetracycline antibiotic with anti-inflammatory and anti-apoptotic properties, has been proposed as a protective agent in diabetes. However, there are no reported studies evaluating the therapeutic efficacy of minocycline in T1D and T2D models for all microvascular complications (DPN, DR, and DKD). Therefore, we performed metabolic profiling in streptozotocin-induced T1D and db/ $\mathrm{db}$ T2D models and compared the efficacy of minocycline in preventing complications to that of insulin and pioglitazone in both models. Minocycline partially ameliorated DR and DKD in T1D and T2D animals, but was less effective than insulin or pioglitazone, and failed to improve DPN in either model. These results suggest that minocycline is unlikely to improve outcomes beyond that achieved with current available therapies in patients with T1D or T2D associated microvascular complications.
\end{abstract}

Abbreviations: DKD: Diabetic kidney disease; DPN: Diabetic peripheral neuropathy; DR: Diabetic retinopathy; STZ: Streptozotocin; T1D: Type 1 diabetes; T2D: Type 2 diabetes.

\section{Introduction}

Both type 1 diabetes (T1D) and type 2 diabetes (T2D) are associated with complications including diabetic peripheral neuropathy (DPN), diabetic retinopathy (DR), and diabetic kidney disease (DKD) [1]. Glycemic control is only partially effective for preventing complications [2]. Thus, a better understanding of disease pathophysiology is crucial for developing effective therapies.

Using T1D and T2D mouse models, we and others have explored transcriptomic changes in complication-prone tissues and identified pathogenic pathways, including inflammation [3], oxidative stress [4], apoptosis [5], and mitochondrial dysfunction [6]. These observations defined potential therapeutic targets that may alleviate end-organ damage.

Minocycline, a tetracycline antibiotic, exerts a wide range of biological effects independent of its antimicrobial properties that make it an attractive therapeutic agent for diabetic complications. It has antiinflammatory, anti-apoptotic, and anti-angiogenic actions, as well as neuroprotective effects [7]. It has been reported to improve DPN [8] and neuropathic pain [9], DR $[10,11]$, and DKD $[12,13]$, possibly due to anti-inflammatory, anti-oxidant, and anti-apoptotic actions [10-13].
Yet, there are no reported studies that explore the simultaneous effects of minocycline on all three complications in both T1D and T2D mouse models.

Herein, we evaluated minocycline treatment efficacy on DPN, DR, and DKD in well-established T1D and T2D models in comparison to insulin or pioglitazone, commonly prescribed to diabetic patients (Figure 1). We found that minocycline did not improve DPN, although partially it ameliorated DR and DKD in T1D and T2D animals, respectively.

\section{Materials and methods}

\section{Animals}

Male C57BLKS (BKS) db/+ mice (control) and BKS db/db mice were purchased from Jackson Laboratories (BKS.Cg-Dock7m +/+ Leprdb/J, Stock No: 000642; Bar Harbor, ME) at 4 weeks of age. Animals were maintained in specific-pathogen-free housing provided by the

${ }^{*}$ Correspondence to: Eva L. Feldman, MD, PhD, 5017 AATBSRB, 109 Zina Pitcher Place, Ann Arbor, MI 48109, USA, E-mail: efeldman@umich.edu; efeldman@umich.edu

Key words: diabetes, minocycline, nephropathy, peripheral neuropathy, retinopathy Received: May 21, 2020; Accepted: June 12, 2020; Published: June 16, 2020 
University of Michigan Unit for Laboratory Animal Medicine and given access to water and chow ad libitum. All protocols were carried out in accordance with the guidelines outlined by the Diabetes Complications Consortium (http://www.diacomp.org) and the National Institutes of Health's (NIH) Guide for the Care and Use of Laboratory Animals (8th Edition). All protocols were approved by the University of Michigan's Institutional Animal Care and Use Committee.

Animal models and study design: To induce T1D, 5-week-old db/+ mice were injected intraperitoneally with $50 \mathrm{mg} / \mathrm{kg}$ streptozotocin (STZ, Sigma Aldrich, St Louis, MO) dissolved in citrate buffer ( $\mathrm{pH} 4.5$ ), for 5 consecutive days $(\mathrm{db} /+\mathrm{STZ})$ [14]. Leptin signaling deficient $\mathrm{db} /$ $\mathrm{db}$ mice were used as a T2D model [14]. Diabetes was defined as fasting blood glucose (FBG) levels over $300 \mathrm{mg} / \mathrm{dL}$ [15]. Starting at 6 weeks of age, mice were randomly assigned to minocycline $(\mathrm{db} /+\mathrm{MINO}, \mathrm{db} /+$ STZ MINO, db/db MINO) or pioglitazone (db/db PIO) (Figure 1) treatment groups and fed AIN-76A standard chow supplemented with $55 \mathrm{mg} / \mathrm{kg} /$ day minocycline or $15 \mathrm{mg} / \mathrm{kg} /$ day pioglitazone, respectively. Minocycline and pioglitazone were both purchased from Michigan Medicine, University of Michigan, and compounded into AIN-76A by Research Diets (New Brunswick, NJ). All other groups were maintained on a standard chow diet (untreated). Insulin was administered via LinBit (both from LinShin, Toronto, Canada) according to the manufacturer's instructions to the $\mathrm{db} /+\mathrm{STZ}$ insulin group starting at 6 weeks of age, with implants replaced every 4 weeks until study termination (i.e., at 10 and 14 weeks). The study duration was 10 weeks and ended when the animals were aged 16 weeks. After phenotyping for each complication, mice were euthanized with $150 \mathrm{mg} / \mathrm{kg}$ sodium pentobarbital administered intraperitoneally. Blood was collected from the superior vena cava for glycated hemoglobin (\%HbAlc) analysis and plasma processing. Hind paws were isolated for intraepidermal nerve fiber density (IENFD) analysis.

Metabolic phenotyping: Animals were weighed every 2 weeks. Fasting blood glucose (FBG) was measured using an AlphaTRAK glucometer (Zoetis, Parsippany-Troy Hills, NJ) every 2 weeks throughout the experimental period. \%HbA1c was measured by ELISA at study termination using the manufacturer's instructions (Mouse Hemoglobin A1c Assay Kit, cat\# 80310; CrystalChem, Elk Grove Village, IL). Serum lipid profiles were analyzed by the Mouse Metabolic Phenotyping Center (MMPC) at Cincinnati (University of Cincinnati Medical Center, OH, USA; www.mmpc.org).

DPN phenotyping: DPN was measured using the protocols outlined by the Diabetes Complications Consortium (www.diacomp.org/shared/ protocols.aspx). Nerve conduction velocities (NCVs) were assessed as a measure of large-fiber nerve function in sural sensory and sciatic-tibial motor nerves at the termination of the study as previously described [16]. IENFD is an anatomical measure of small nerve fibers. Fixed hind paw plantar tissue was stained with a pan-axonal marker, ubiquitin C-terminal hydrolase L1 (UCHL1; 1:2000; Proteintech, Rosemont, IL) [17]. Three random fields per mouse were imaged $(1024 \times 1024$ pixel resolution, $3.3 \mu \mathrm{m}$ optical section thickness, FluoView 500 confocal microscope, $20 \times 1.2$ objective, Olympus, Tokyo, Japan). MetaMorph (Molecular Devices, San Jose, CA) flattened ten images per stack, and IENFD was represented as fiber counts per $\mathrm{mm}$.

DR phenotyping: DR was evaluated using protocols from the Diabetes Complications Consortium. Briefly, retinal DNA fragmentation was measured at the study termination by apoptotic DNA cleavage ELISA (Cell Death Detection, Roche Applied Science, Indianapolis, IN) and normalized to retinal wet weight [18].
DKD phenotyping: DKD was assessed using protocols from the Diabetes Complications Consortium and as previously reported [19]. Urinary albumin-to-creatinine ratio (ACR) was measured in mice placed in metabolic cages for the last $72 \mathrm{~h}$ of the study. Urine from the final $24 \mathrm{~h}$-period was collected and analyzed for albumin and creatinine using the Albuwell $\mathrm{M}$ and Creatinine Companion assays (Exocell, Philadelphia, PA) [20]. To assess glomerular hypertrophy, perfused left kidney tissue was fixed in paraformaldehyde, paraffin-embedded, sectioned to $3 \mu \mathrm{m}$ thickness, and stained with periodic acid-Schiff (PAS) stain [19]. Fifteen glomerular tufts per mouse were randomly chosen for hypertrophy analysis. Mesangial index was determined by calculating the PAS-positive area of each glomerulus relative to total area. MetaMorph was used for quantification of glomerular and PASpositive areas and microscope images were captured using a digital camera, as per our published protocol [19].

\section{Statistical analyses}

Statistical analyses were performed using GraphPad Prism 7 [21]. Normality of data was determined using Brown-Forsythe F-tests. Statistically significant differences $(\mathrm{P}<0.05)$ for normally distributed data were analyzed using one-way ANOVA followed by Tukey's posttest for multiple comparisons. Datasets were $\log 2$ transformed for non-normally distributed data, and the Brown-Forsythe F-test rerun. When $\log 2$-transformation normalized distribution, a one-way ANOVA followed by Tukey's post-test for multiple comparisons was used. When $\log 2$-transformation did not normalize distribution, the non-parametric Kruskal-Wallis test, with Dunn's post-test for multiple comparisons was used on the original, non-transformed dataset. Data are presented as mean \pm standard error of the mean (SEM).

\section{Results}

Minocycline only marginally affects metabolic phenotypes in T1D and T2D mice. Throughout the study, T1D db/+ STZ mice showed significant weight loss (Figures 2A and 2C) and elevated FBG (Figures $3 \mathrm{~A}-3 \mathrm{C})$ relative to nondiabetic controls $(\mathrm{db} /+)$. At study termination, the higher FBG was reflected by increased \%HbAlc in T1D db/+ STZ mice versus controls (Figures $3 \mathrm{C}$ and $3 \mathrm{D}$ ). The lipid profile of $\mathrm{T} 1 \mathrm{D} \mathrm{db} /+$ STZ mice was broadly similar to $\mathrm{db} /+$ controls, except they exhibited higher cholesterol levels, without differences in triglycerides, non-esterified fatty acids (NEFAs), and phospholipids (Figure 4). Minocycline did not affect body weight (Figure 2C), terminal \%HbA1c (Figure 3D), or lipid profiles (Figure 4) in T1D db/+ STZ mice, although terminal FBG was marginally, though significantly, higher versus controls (Figure 3C). Minocycline also had no effect on control $\mathrm{db} /+$ mice. In contrast, insulin partially restored terminal body weight (Figure 2C), FBG, and \%HbAlc (Figures 3C and 3D) in T1D db/+ STZ mice relative to controls. It also significantly reduced triglycerides and NEFAs, but not cholesterol or phospholipids levels in T1D db/+ STZ mice versus controls (Figure 4).

During the experimental period, T2D db/db mice exhibited significant weight gain (Figures $2 \mathrm{~B}$ and $2 \mathrm{C}$ ) and raised FBG (Figures $3 \mathrm{~B}$ and $3 \mathrm{C}$ ) relative to control $\mathrm{db} /+$ animals. At study conclusion, the higher FBG was paralleled by an elevated $\% \mathrm{HbA} 1 \mathrm{c}$ in $\mathrm{T} 2 \mathrm{D} \mathrm{db} / \mathrm{db}$ mice versus controls (Figures $3 \mathrm{C}$ and $3 \mathrm{D}$ ). T2D db/db mice had profound dyslipidemia, with increased cholesterol, NEFAs, and phospholipids, but unaltered triglycerides in comparison to controls (Figure 4). Minocycline slightly reduced terminal body weight (Figure 2C) but did not improve terminal glycemic control in $\mathrm{T} 2 \mathrm{D} \mathrm{db} / \mathrm{db}$ mice (Figures $3 \mathrm{C}$ and 3D). It also slightly but not significantly perturbed NEFAs in T2D $\mathrm{db} / \mathrm{db}$ mice (Figure $4 \mathrm{C}$ ). Otherwise, minocycline was without effect 
Strain: Male BKS.Cg-m +/+ Leprdb/J

$\square$ Standard Diet

$\square$ Minocycline: $55 \mathrm{mg} / \mathrm{kg} / \mathrm{d}$

STZ i.p. $5 \times 50 \mathrm{mg} / \mathrm{kg}$

Pioglitazone: $15 \mathrm{mg} / \mathrm{kg} / \mathrm{d}$

\begin{tabular}{|l|l|}
\hline \multicolumn{2}{|c|}{$d b /+$ standard diet } \\
\hline \hline & $d b /+$ minocycline \\
\hline
\end{tabular}
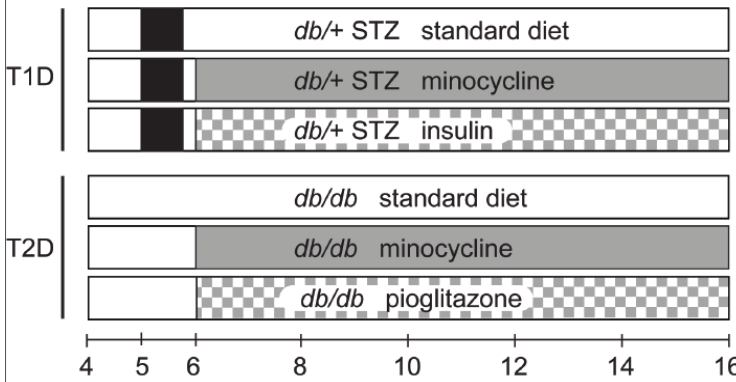

Figure 1. Study design. The study consisted of eight groups: (i) untreated $d b /+$ controls, (ii) minocycline-treated $d b /+$ controls, (iii) untreated T1D $d b /+$ STZ mice, (iv) minocycline-treated T1D $d b /+$ STZ mice, (v) insulin-treated T1D $d b /+$ STZ mice, (vi) untreated T2D $d b / d b$ mice, (vii) minocycline-treated T2D $d b / d b$ mice, and (viii) pioglitazone-treated T2D $d b / d b$ mice. Untreated cohorts just ate standard chow. Minocycline dose was $55 \mathrm{mg} / \mathrm{kg} /$ day, and pioglitazone dose was $15 \mathrm{mg} / \mathrm{kg} / \mathrm{day}$. Treatment started in mice aged 6 weeks and lasted $10 \mathrm{weeks}$ till study conclusion when mice were aged 16 weeks. Body weight and fasting blood glucose (FBG) levels were recorded every two weeks. At 16 weeks, metabolic, DPN, DR, and DKD phenotyping was performed

a
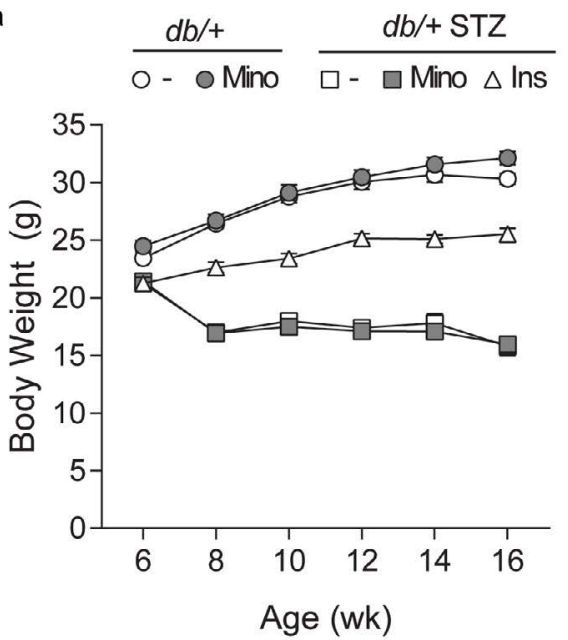
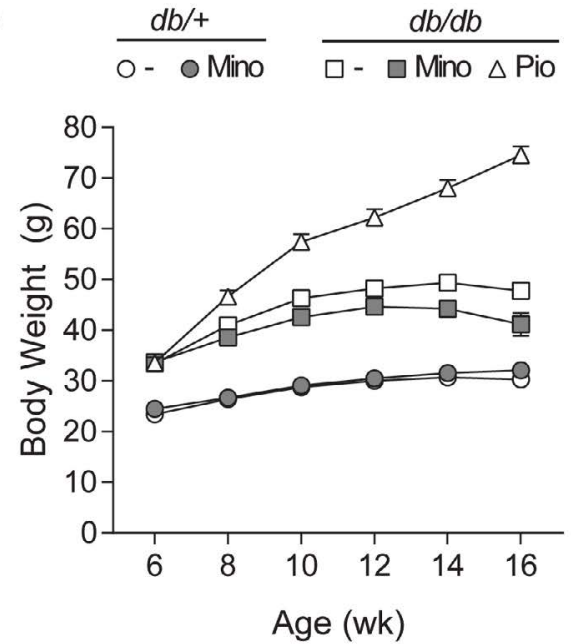

C

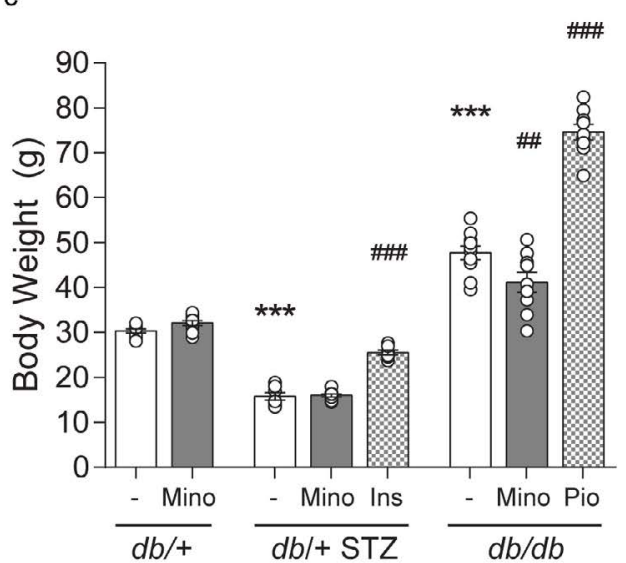

Figure 2. Longitudinal and terminal body weight. Body weights were measured at $6,8,10,12,14$, and 16 weeks and are shown for control $d b /+$ and $d b /+$ MINO against: (A) T1D $d b /+$ STZ, T1D $d b /+$ STZ MINO, T1D $d b /+$ STZ INS, and (B) T2D $d b / d b$, T2D $d b / d b$ MINO, T2D $d b / d b$ PIO. (C) Terminal body weights measured at 16 weeks. INS, insulin; MINO, minocycline;

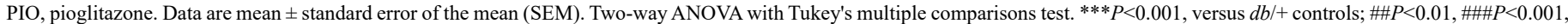
versus T1D $d b /+\mathrm{STZ}$ or versus T2D $d b / d b ; \mathrm{n}=7-10$ mice 

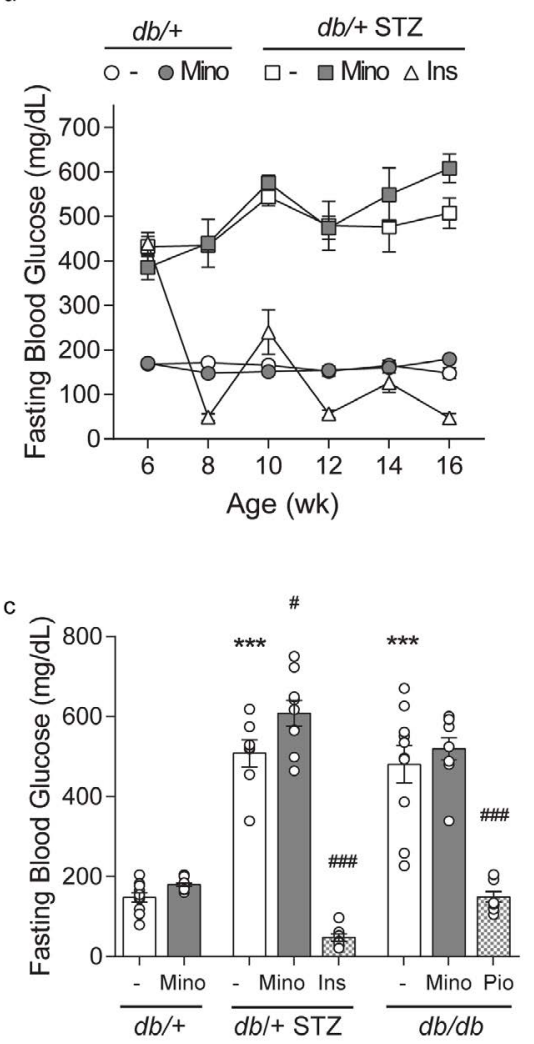
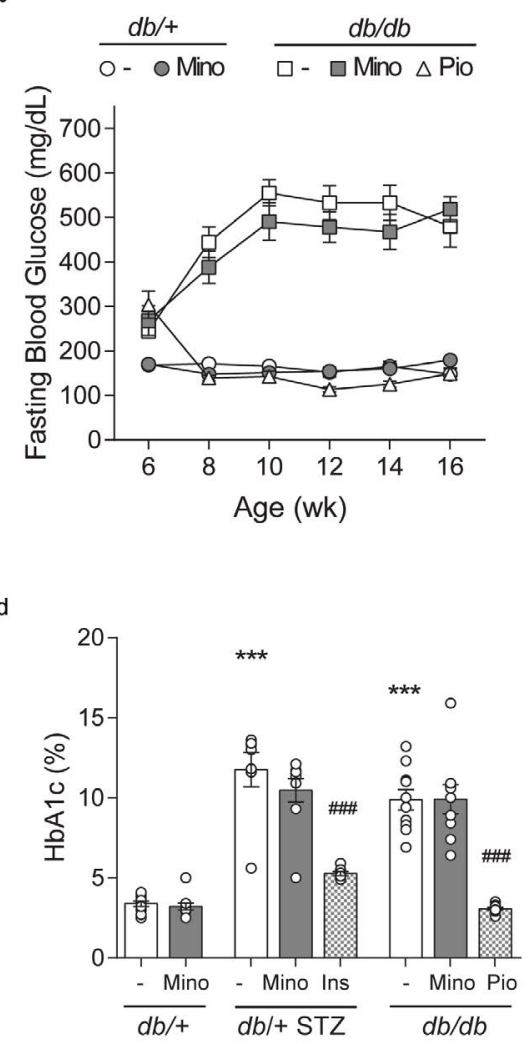

Figure 3. Longitudinal and terminal glycemic status. FBG was recorded at $6,8,10,12,14$, and 16 weeks and is shown for control $d b /+$ and $d b /+$ MINO against: (A) T1D $d b /+$ STZ, T1D $d b /+$ STZ MINO, T1D $d b /+$ STZ INSULIN, and (B) T2D $d b / d b$, T2D $d b / d b$ MINO, T2D $d b / d b$ PIO. Terminal (C) body weights and (D) percent glycated hemoglobin (\%HbA1c) were measured at 16 weeks. INS, insulin; MINO, minocycline; PIO, pioglitazone. Data are mean \pm SEM. Two-way ANOVA with Tukey's multiple comparisons test. $* * * P<0.001$, versus $d b /+$ controls; $\# P<0.05, \# \# P<0.001$, versus T1D $d b /+\mathrm{STZ}$ or versus $\mathrm{T} 2 \mathrm{D} d b / d b ; \mathrm{n}=7-10$ mice
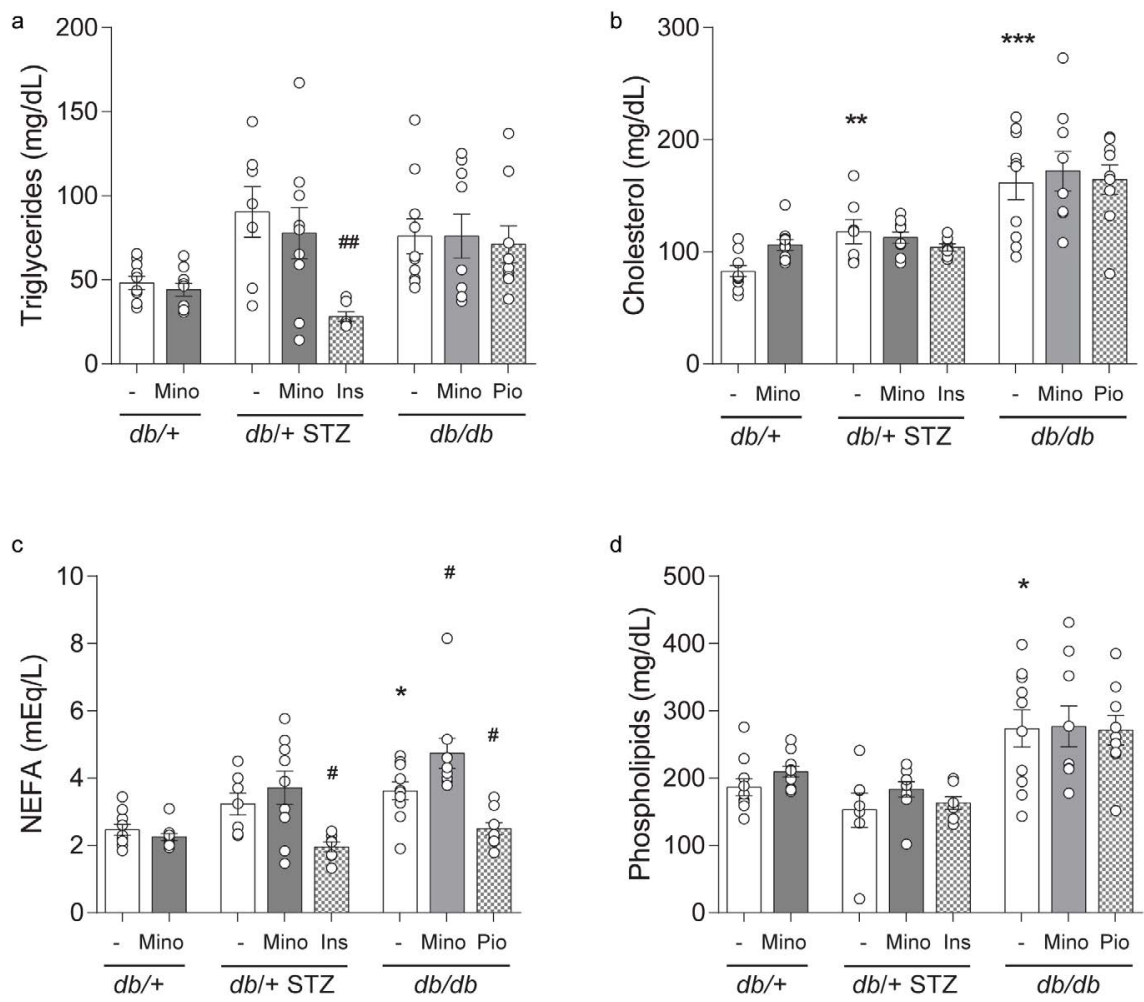

Figure 4 Terminal lipid profiles. (A) Triglycerides, (B) cholesterol, (C) non-esterified fatty acids (NEFAs), and (D) phospholipids were measured for all eight mouse cohorts at 16 weeks. INS, insulin; MINO, minocycline; PIO, pioglitazone. Data are mean \pm SEM. Two-way ANOVA with Tukey's multiple comparisons test. $* P<0.05, * * P<0.01, * * * P<0.001$, versus $d b /+$ controls; $\# P<0.05, \# \# P<0.01$, versus T1D $d b /+\mathrm{STZ}$ or versus T2D $d b / d b ; \mathrm{n}=7-10$ mice 
on the metabolic profile in $\mathrm{T} 2 \mathrm{D} \mathrm{db} / \mathrm{db}$ animals. On the other hand, pioglitazone exerted a greater influence on $\mathrm{T} 2 \mathrm{D} \mathrm{db} / \mathrm{db}$ mice, markedly raising their weight (Figures $2 \mathrm{~B}$ and $2 \mathrm{C}$ ) but lowering their terminal FBG and \%HbAlc to db/+ levels (Figures 3C and 3D). Pioglitazone also ameliorated NEFAs in T2D db/db mice but did not impact cholesterol or phospholipids (Figure 4). Overall, minocycline only marginally affected metabolic phenotypes in diabetic mice, whereas insulin and pioglitazone significantly improved multiple metabolic criteria in T1D and $\mathrm{T} 2 \mathrm{D}$ mice, respectively.

\section{Minocycline does not improve DPN phenotypes in T1D and T2D mice}

STZ-induced T1D db/+ mice display microvascular complications, including DPN, DR, and DKD [14,22,23]. As anticipated, T1D db/+ STZ mice had significantly decreased large-fiber sensory and motor NCVs relative to control $\mathrm{db} /+$ animals, although their small-fiber IENFD only trended towards lower values $(\mathrm{P}=0.052$; Figures $5 \mathrm{~A}-5 \mathrm{C})$. Minocycline had no effect on any of these DPN metrics, in either T1D $\mathrm{db} /+\mathrm{STZ}$ or control $\mathrm{db} /+$ mice. In contrast, insulin normalized large- fiber conduction and increased IENFD in T1D STZ $\mathrm{db} /+$ mice relative to controls (Figures 5A-5C). We have previously reported that obese, leptin signaling-deficient T2D db/db mouse develops DPN $[3,20]$. As predicted, $\mathrm{T} 2 \mathrm{D} \mathrm{db} / \mathrm{db}$ animals in this study displayed pronounced neuropathy in both large- and small-fiber measures versus controls (Figures 5A-5C). Minocycline did not affect the DPN phenotype in these T2D animals. However, pioglitazone did exert a significant effect on large-fiber NCVs. In summary, minocycline does not appear to improve DPN in diabetic mice, while insulin and pioglitazone significantly improved DPN phenotypes in T1D and T2D mice, respectively.

\section{Minocycline improves DR in T1D and DKD in T2D mice}

Retinal apoptosis is a surrogate for DR progression, when quantified relative to wet retinal tissue weight. As anticipated, retinal apoptosis was increased in T1D db/+ STZ mice in relation to controls (Figure 5D). Minocycline lowered retinal apoptosis significantly in T1D db/+ STZ mice, as did insulin administration. Increased apoptosis was also observed in the retina of the $\mathrm{T} 2 \mathrm{D} \mathrm{db} / \mathrm{db}$ mice (Figure $5 \mathrm{D}$ ), which was a

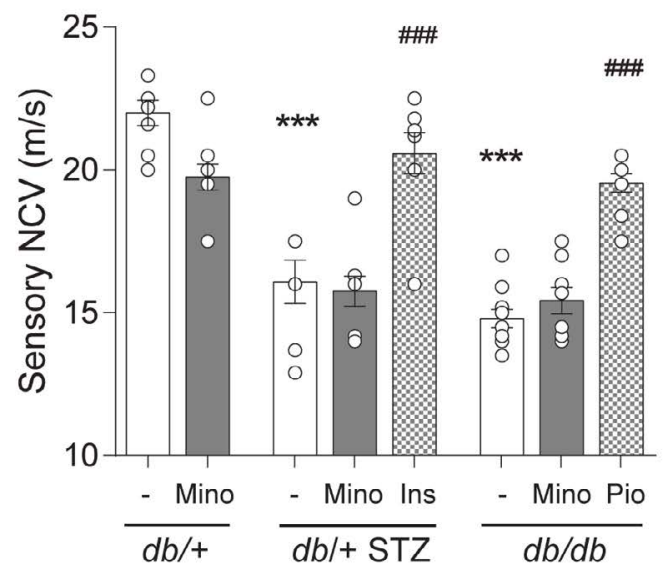

C

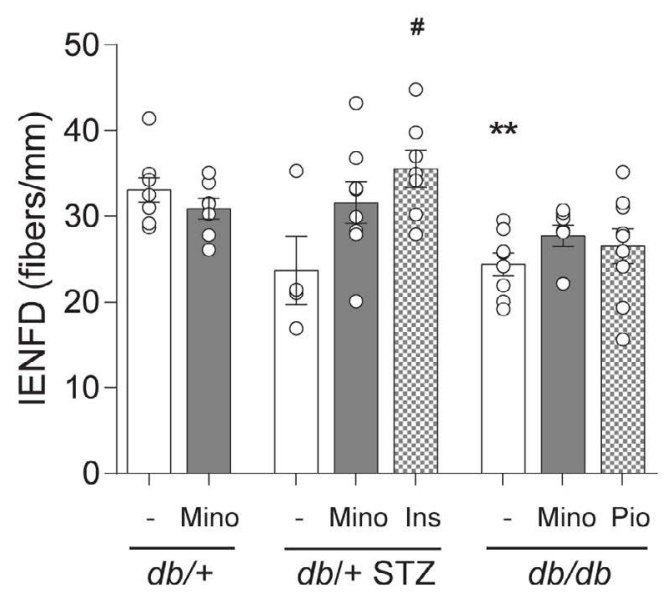

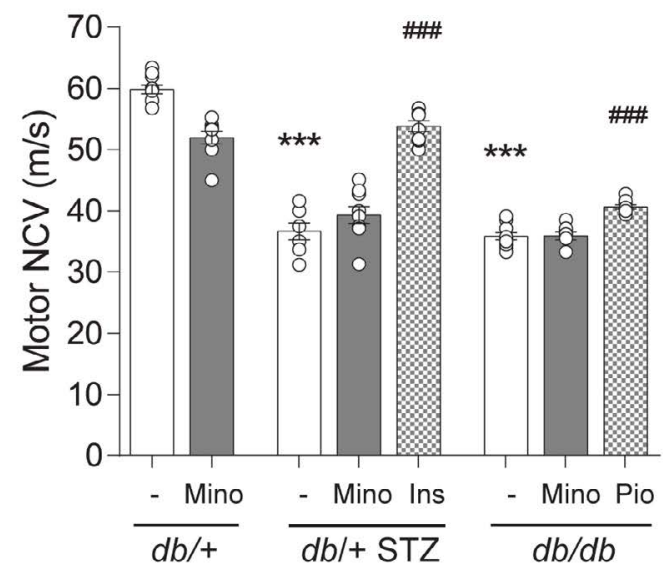

d

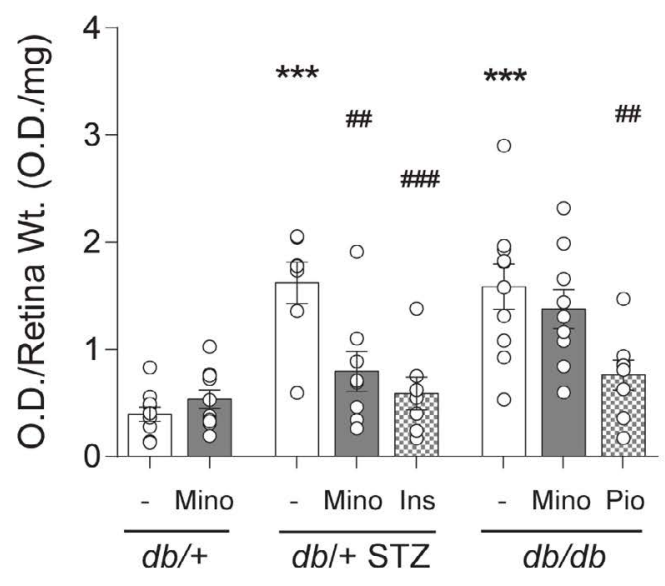

Figure 5. Terminal DPN and DR phenotyping. Terminal large-fiber nerve conduction velocities (NCVs) in (A) sural sensory and (B) sciatic motor nerves. (C) Terminal small-fiber intraepidermal nerve fiber density (IENFD). (D) Terminal apoptosis by retinal DNA fragmentation expressed as optical density from ELISA relative to wet retinal tissue weight. All metrics were measured for all eight mouse cohorts at 16 weeks. INS, insulin; MINO, minocycline; PIO, pioglitazone. Data are mean \pm SEM. Two-way ANOVA with Tukey's multiple comparisons

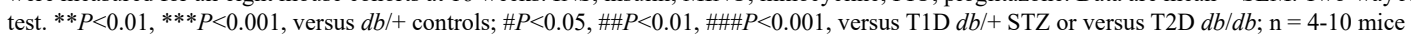



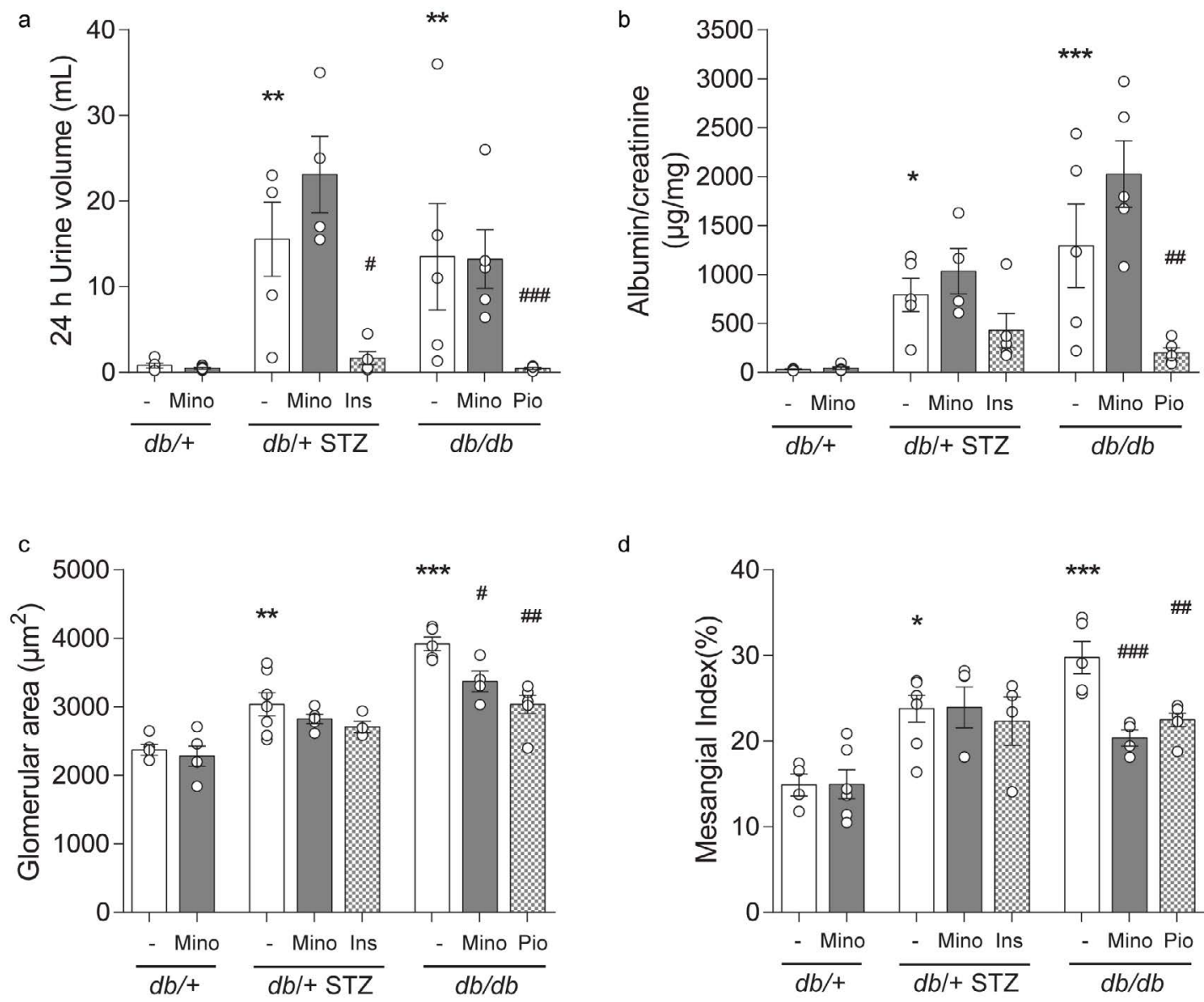

d

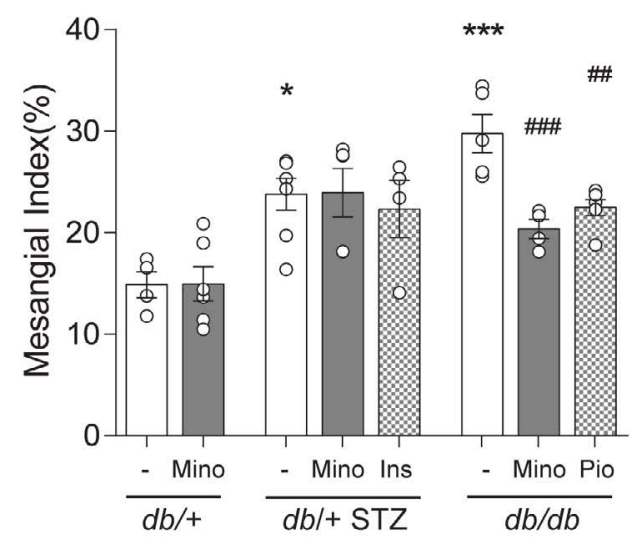

Figure 6. Terminal DKD phenotyping. Terminal metrics of renal function by (A) $24 \mathrm{~h}$ urine volume and (B) albumin-to-creatinine ratio. Terminal metrics of renal histopathology by (C) glomerular area and (D) mesangial index. All metrics were measured for all eight mouse cohorts at 16 weeks. INS, insulin; MINO, minocycline; PIO, pioglitazone. Data are mean \pm SEM.

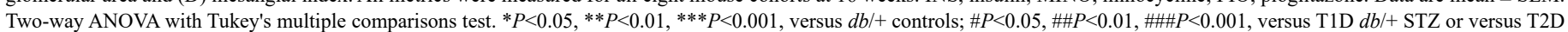
$d b / d b ; \mathrm{n}=4-7$ mice

ameliorated by pioglitazone but not by minocycline treatment. Thus, minocycline improved DR phenotype in the T1D but not the T2D mouse model.

Both T1D db/+ STZ and T2D db/db mice exhibited elevated ACR compared to $\mathrm{db} /+$ controls (Figures $6 \mathrm{~A}$ and $6 \mathrm{~B}$ ), as well as increased glomerular area and mesangial index (Figures 6A and 6B). Minocycline had no effect on any of these parameters in T1D db/+ STZ mice but partially prevented glomerular hypertrophy and increased mesangial index in the $\mathrm{T} 2 \mathrm{D} \mathrm{db} / \mathrm{db}$ animals. Minocycline did not affect ACR in the $\mathrm{T} 2 \mathrm{D} \mathrm{db} / \mathrm{db}$ mice, while pioglitazone treatment prevented all aspects of DKD in the T2D model.

\section{Discussion}

Multiple studies identify inflammation, oxidative stress, apoptosis, and dysregulated immunity and energy metabolism as consistent features in nerve, retinal, and kidney tissue in both mouse models and patients with T1D and T2D [3-6,24-32]. We and others [8-13,33-36] have consequently investigated minocycline, with its anti-inflammatory, anti-apoptotic, and anti-oxidant effects [7], as a possible therapeutic agent in DPN, DR, and DKD. Despite several reports showing that minocycline improved aspects of each individual complication $[8,10$ 13,34-36], the current study represents the first time the simultaneous effects of minocycline on DPN, DR, and DKD were compared in a
STZ-induced T1D model and a leptin signaling-deficient T2D model on a BKS background. We also contrasted the therapeutic efficacy of minocycline to insulin and pioglitazone in T1D db/+ STZ and T2D db/ $\mathrm{db}$ mice, respectively.

By ten weeks into the study, T1D and T2D animals exhibited severe diabetes, including elevation in terminal FBG and \%HbAlc (Figure 3 ), and perturbations in serum lipid profiles, particularly in the T2D animals (Figure 4). Minocycline did not affect body weight and FBG in our experimental T1D and T2D cohorts and had little effect on blood lipids which is consistent with previous reports in T1D rat $[9,11,34,36,37]$ and T2D mouse [13] models and in humans [38]. Although we did not anticipate that minocycline would impact metabolic parameters, we predicted that it would prevent diabetic complications due to its antiinflammatory, anti-apoptotic, and anti-oxidant effects [7]. Minocycline also possesses favorable pharmacokinetic (PK) properties, including good nervous system penetration and long serum half-life in humans [39]. However, the protective effects of minocycline on diabetic microvascular complications were modest, at best, and varied between diabetic models.

In our study, a 10-week regimen of minocycline ( $55 \mathrm{mg} / \mathrm{kg}$ in chow) had no effect on NCVs or IENFD, large- and small-fiber measures of DPN respectively in T1D db/+ STZ and T2D db/db animals (Figures 5A-5C). In agreement with our findings, $25 \mathrm{mg} / \mathrm{kg}$ minocycline daily for 
8 weeks yielded no effects on NCV or IENFD deficits in STZ-induced T1D rats [37]. In contrast, minocycline improved sural NCVs and pain related behaviors at a dose of $40 \mathrm{mg} / \mathrm{kg}$ for 3 days [8]. Similar reports of minocycline efficacy on pain are reported after short treatment trials $[9,33,40]$, albeit intrathecal minocycline $(10 \mathrm{nmol})$ did not ameliorate mechanical allodynia, as assessed by Frey monofilament in 10-weekold mice [41]. While data are limited, a 6-week trial of minocycline (100 $\mathrm{mg}$, twice daily versus placebo) in $50 \mathrm{~T} 2 \mathrm{D}$ subjects with DPN resulted in improved vibratory sensation and decreased pain perception in both minocycline treated and placebo subjects, although the improvement was greater in the minocycline arm [38]. A caveat of this trial is that quantitative NCVs and IENFD were not assessed, a requirement for demonstrating drug efficacy in clinical trials of DPN according to the Toronto Expert Panel on DPN [42]. The variation in the mouse studies, and the results of this sole clinical trial, suggest a possible short-lived salutary effect of minocycline. Clearly, further studies in both mice and man are needed to definitively determine any long-term benefit of minocycline treatment. In contrast, insulin completely normalized small- and large-fiber DPN metrics in T1D db/+ STZ mice (Figures 5A5C), as anticipated [2]. Surprisingly, however, pioglitazone improved large-fiber NCVs but not small-fiber IENFDs in T2D db/+ animals (Figures 5A-5C), an effect opposite to our previous observations in the same T2D model [26]. We anticipate this discrepancy between studies is related to a variation in gut microbiota that can affect the metabolic phenotype [43] and hence DPN, a possibility we are currently examining.

In the current study, minocycline decreased DNA fragmentation, a surrogate marker of $\mathrm{DR}$, in the retina of T1D db/+ STZ but not of $\mathrm{T} 2 \mathrm{D} \mathrm{db} / \mathrm{db}$ animals (Figure 5D). Our results agree with the positive effects of minocycline treatment on DR in a T1D STZ rat model dosed twice daily with minocycline $(22.5 \mathrm{mg} / \mathrm{kg})$ for 10 days [34], as well as T1D STZ rats treated with 2.5 or $5 \mathrm{mg} / \mathrm{kg}$ minocycline daily for 4 or 8 weeks [10,11], or $50 \mathrm{mg} / \mathrm{kg}$ daily for 4 weeks [35]. There was no effect of minocycline on $\mathrm{T} 2 \mathrm{D} \mathrm{db} / \mathrm{db}$ mice (Figure $5 \mathrm{D}$ ), and to our knowledge, there are no reports of minocycline treatment in T2D mouse models for comparison. We did observe that both insulin and pioglitazone lowered retinal apoptosis in T2D $\mathrm{db} / \mathrm{db}$ group and T1D $\mathrm{db} /+\mathrm{STZ}$ animals, respectively, a result that was anticipated for the T1D model [44] (Figure 5D). We speculate the efficacy of minocycline in our T1D but not T2D cohorts is intrinsic to the type of diabetes. As with DPN, further studies are needed to confirm our single report on the lack of minocycline efficacy on DR in T2D and explore its differential effects in T1D and T2D.

In our study, the effect of minocycline on DKD progression was distinct in each model (Figure 6), with improvement in some aspects of $\mathrm{DKD}$ in T2D db/db mice, but without effect in T1D db/+ STZ animals. In agreement with our results, STZ-induced T1D rats receiving daily minocycline $(50 \mathrm{mg} / \mathrm{kg})$ for 4 weeks had no improvement in renal histological markers, as assessed by renal hypertrophy and tubulointerstitial fibrosis index [36]. In contrast, another study in T1D STZ rats that received $20 \mathrm{mg} / \mathrm{kg}$ minocycline daily for 4 or 16 weeks found a reduction in proteinuria [12], with lowering of renal apoptosis and glomerulosclerosis, although no change in glomerular area was noted. In contrast to our results in T1D, treatment of T2D $\mathrm{db} / \mathrm{db}$ mice with minocycline partly prevented glomerular hypertrophy and mesangial expansion but did not affect ACR (Figures 6A and 6B). Our data agree with a parallel study on the T2D db/db model, where a 12 -week regimen of daily minocycline $(5 \mathrm{mg} / \mathrm{kg})$ in mice aged 8 to
20 weeks improved renal histology (apoptosis by TUNEL, fractional mesangial area) [13]. Our data also agree with two human clinical trials, where minocycline treatment had no effect on ACR [38]. Thus, the clinical trials and our study suggest that minocycline has an effect on glomerular growth and mesangial matrix expansion but no effect on albuminuria. Specifically, minocycline may have a specific therapeutic effect in T2D on glomerular mesangial cells and/or glomerular macrophages that play a direct role in mesangial expansion, but little or no effect on podocyte alterations in early diabetes, changes that are generally manifested by albuminuria.

\section{Conclusion}

In summary, the literature is inconsistent on the potential effects of minocycline on diabetic microvascular complications. Differences could arise from variation in rodent models (rat versus mouse), drug dose and therapy duration, disease duration and age of animals at treatment commencement, as well as type of diabetes. Our study determined the efficacy of minocycline in a side-by-side comparison in T1D and T2D mice on the same genetic background. We found that minocycline has some potential for ameliorating microvascular complications in T1D and T2D, with improvement in DR in the T1D mice and DKD in the T2D mice, with no discernible effect on DPN in either model. While glycemic control via insulin or pioglitazone in T1D and T2D cohorts, respectively, rescued microvascular complications to a greater extent than minocycline, there remains the possibility patients could receive potential benefit from adjunctive minocycline therapy, i.e., it is possible that minocycline could exert a greater effect over and above good glycemic/metabolic control. Minocycline was well-tolerated with few adverse events in both our study as well as the other reported rodent [8,10-13,34-36] and human studies [38,45-47], supporting its further study in well established, well phenotyped animal models of diabetes, as well as in man.

\section{Funding}

This research work used the Cincinnati MMPC for serum lipid profiles measurements (DK059630) and Michigan MMPC for DPN phenotyping (U2CDK110768). Funding was provided by the National Institutes of Health (1R24082841 to E.L.F., S.F.A., F.C.B. and S.P.; U2CDK110768 and R21NS102924 to E.L.F.; R01EY020582, R01EY029349 and R01EY020823 to S.F.A.; P30EY007003 Kellogg Eye Center Core Center for Vision Research; P30DK081943, and P30DK89503 to S.P.); Novo Nordisk Foundation (NNF14OC0011633 to E.L.F.); Milstein, Nathan and Rose Research Fund (to S.A.E.); NeuroNetwork for Emerging Therapies and the A. Alfred Taubman Medical Research Institute (to S.A.E. and E.L.F.).

\section{Author contributions}

S.A.E., P.D.O., L.M.H., and E.L.F. designed the studies. S.A.E., P.D.O., L.M.H., J.M.H., F.E.M., and H.Z. conducted the studies. S.A.E., P.D.O., L.M.H., S.F.A., F.C.B., S.P., and E.L.F. analyzed the data. S.A.E., S.N., M.G.S., and E.L.F. wrote the manuscript. All authors approved the final version.

\section{Acknowledgements}

The authors acknowledge the technical expertise of Ms. Crystal Pacut, at the University of Michigan in performing ELISAs. They also thank Dr. Stacey Sakowski Jacoby for expert editorial assistance. 


\section{Competing interest}

P.D.O. is currently employed by Eli Lilly, but all contributions made by P.D.O. occurred while he was employed by the University of Michigan. Eli Lilly was not involved in any way with the research presented herein.

\section{References}

1. Zheng Y, Ley SH, Hu FB (2018) Global aetiology and epidemiology of type 2 diabetes mellitus and its complications. Nat Rev Endocrinol 14: 88-98. [Crossref]

2. Callaghan BC (2012) Enhanced glucose control for preventing and treating diabetic neuropathy. Cochrane Database Syst Rev.

3. Hinder LM (2018) Transcriptional networks of progressive diabetic peripheral neuropathy in the $\mathrm{db} / \mathrm{db}$ mouse model of type 2 diabetes: An inflammatory story. Exp Neurol 305: 33-43.

4. Bogdanov P (2014) The $\mathrm{db} / \mathrm{db}$ mouse: a useful model for the study of diabetic retinal neurodegeneration. PLoS One 9: e97302.

5. Pande M (2011) Transcriptional Profiling of Diabetic Neuropathy in the BKS $d b / d b$ Mouse: A Model of Type 2 Diabetes. Diabetes 60: 1981-1989. [Crossref]

6. Sas KM (2016) Tissue-specific metabolic reprogramming drives nutrient flux in diabetic complications. JCI Insight 1: e86976.

7. Garrido-Mesa N, Zarzuelo A, Galvez J (2013) Minocycline: far beyond an antibiotic. Br J Pharmacol 169: 337-352.

8. Yang C (2017) Minocycline attenuates the development of diabetic neuropathy by inhibiting spinal cord Notch signaling in rat. Biomed Pharmacother 94: 380-385.

9. Pabreja K (2011) Minocycline attenuates the development of diabetic neuropathic pain: possible anti-inflammatory and anti-oxidant mechanisms. Eur J Pharmacol 661: 15-21.

10. Chen W (2017) Activation of the TXNIP/NLRP3 inflammasome pathway contributes to inflammation in diabetic retinopathy: a novel inhibitory effect of minocycline. Inflamm Res 66: 157-166.

11. Wu Y (2015) Minocycline inhibits PARP1 expression and decreases apoptosis in diabetic retinopathy. Mol Med Rep 12: 4887-4894. [Crossref]

12. Yuan H (2016) Minocycline attenuates kidney injury in a rat model of streptozotocininduced diabetic nephropathy. Biol Pharm Bull 39: 1231-1237.

13. Shahzad K (2016) Stabilization of endogenous Nrf2 by minocycline protects against Nlrp3-inflammasome induced diabetic nephropathy. Sci Rep 6: 34228 .

14. O'Brien PD, Sakowski SA, Feldman EL (2014) Mouse models of diabetic neuropathy. ILAR J 54: 259-272.

15. Leiter EH (2009) Selecting the "right" mouse model for metabolic syndrome and type 2 diabetes research. Methods Mol Biol 560: 1-17.

16. Oh SS (2010) The effects of anesthesia on measures of nerve conduction velocity in male C57B16/J mice. Neurosci Lett 483: 127-131.

17. Cheng HT (2012) Nerve growth factor/p38 signaling increases intraepidermal nerve fiber densities in painful neuropathy of type 2 diabetes. Neurobiol Dis 45: 280-287.

18. Abcouwer SF (2010) Effects of ischemic preconditioning and bevacizumab on apoptosis and vascular permeability following retinal ischemia-reperfusion injury. Invest Ophthalmol Vis Sci 51: 5920-5933.[Crossref]

19. Zhang H (2008) Rosiglitazone reduces renal and plasma markers of oxidative injury and reverses urinary metabolite abnormalities in the amelioration of diabetic nephropathy. Am J Physiol Renal Physiol 295: F1071- F1081.

20. Hinder LM (2019) Mitochondrial uncoupling has no effect on microvascular complications in type 2 diabetes. Sci Rep 9: 881.

21. Festing MF, Altman DG (2002) Guidelines for the design and statistical analysis of experiments using laboratory animals. Ilar $j$ 43: 244-258.

22. Azushima K, Gurley SB, Coffman TM (2018) Modelling diabetic nephropathy in mice. Nat Rev Nephrol 14: 48-56.

23. Olivares AM (2017) Animal models of diabetic retinopathy. Curr Diab Rep 17: 93.

24. Hinder LM (2017) Comparative RNA-Seq transcriptome analyses reveal distinct metabolic pathways in diabetic nerve and kidney disease. $J$ Cell Mol Med 21: 21402152. [Crossref]
25. Hur J (2016) Transcriptional networks of murine diabetic peripheral neuropathy and nephropathy: common and distinct gene expression patterns. Diabetologia 59: 297306 .

26. Hur J (2015) The metabolic syndrome and microvascular complications in a murine model of type 2 diabetes. Diabetes 64: 3294-304.

27. McGregor BA (2018) Conserved transcriptional signatures in human and murine diabetic peripheral neuropathy. Scientific Reports 8: 17678.

28. O'Brien PD (2015) BTBR ob/ob mice as a novel diabetic neuropathy model: neurological characterization and gene expression analyses. Neurobiology of disease 73: 348-355.

29. Freeman WM (2009) Transcriptomic comparison of the retina in two mouse models of diabetes. J Ocul Biol Dis Infor 2: 202-213.

30. Kandpal RP (2012) Transcriptome analysis using next generation sequencing reveals molecular signatures of diabetic retinopathy and efficacy of candidate drugs. Mol Vis 18: $1123-1146$.

31. Hodgin JB (2013) Identification of cross-species shared transcriptional networks of diabetic nephropathy in human and mouse glomeruli. Diabetes 62: 299-308. [Crossref]

32. Komers R (2014) Transcriptome-based analysis of kidney gene expression changes associated with diabetes in OVE26 mice, in the presence and absence of losartan treatment. PLoS One 9: e96987.

33. Amorim, D (2017Minocycline reduces mechanical allodynia and depressive-like behaviour in type-1 diabetes mellitus in the rat. Behav Brain Res 327: 1-10.

34. Krady JK (2005) Minocycline reduces proinflammatory cytokine expression, microglial activation, and caspase- 3 activation in a rodent model of diabetic retinopathy. Diabetes 54: 1559-65. [Crossref]

35. Bhatt LK, Addepalli V (2010) Attenuation of diabetic retinopathy by enhance inhibition of MMP-2 and MMP-9 using aspirin and minocycline in streptozotocindiabetic rats. Am J Transl Res 2: 181-189.

36. Kumar Bhatt, Addepalli V (2011) Minocycline with aspirin: an approach to attenuate diabetic nephropathy in rats. Ren Fail 33: 72-78

37. Ali S (2014) Matrix metalloproteinase-2 is downregulated in sciatic nerve by streptozotocin induced diabetes and/or treatment with minocycline: Implications for nerve regeneration. Exp Neurol 261: 654-665.

38. Syngle A (2014) Minocycline improves peripheral and autonomic neuropathy in type 2 diabetes: MIND study. Neurol Sci 35: 1067-1073. [Crossref]

39. Cunha BA, Baron J, Cunha CB (2017) Similarities and differences between doxycycline and minocycline: clinical and antimicrobial stewardship considerations. Eur J Clin Microbiol Infect Dis 37: 15-20.

40. Miranda HF (2017) Antinociceptive interaction of gabapentin with minocycline in murine diabetic neuropathy. Inflammopharmacology 25: 91-97.

41. Liao YH (2011) Spinal astrocytic activation contributes to mechanical allodynia in a mouse model of type 2 diabetes. Brain Res 1368: 324-335. [Crossref]

42. Dyck PJ (2011) Diabetic polyneuropathies: update on research definition, diagnostic criteria and estimation of severity. Diabetes Metab Res Rev 27: 620-628.

43. Velagapudi VR (2010) The gut microbiota modulates host energy and lipid metabolism in mice. J Lipid Res 51: 1101-1112.

44. Nathan DM (1993) The effect of intensive treatment of diabetes on the development and progression of long-term complications in insulin-dependent diabetes mellitus. $N$ Engl J Med 329: 977-986. [Crossref]

45. Cukras CA (2012) Oral minocycline for the treatment of diabetic macular edema (DME): results of a phase I/II clinical study. Invest Ophthalmol Vis Sci 53: 3865-3874.

46. Shah AP (2016) Effects of minocycline on urine albumin, interleukin-6, an osteoprotegerin in patients with diabetic nephropathy: A randomized controlled pilot trial. PLoS One 11: e0152357. [Crossref]

47. Liu F (2018) No effect of adjunctive minocycline treatment on body metabolism in patients with schizophrenia. J Clin Psychopharmacol 38: 125-128.

Copyright: (C2020 Eid SA. This is an open-access article distributed under the terms of the Creative Commons Attribution License, which permits unrestricted use, distribution, and reproduction in any medium, provided the original author and source are credited. 